\title{
Towards a Typology of Diagrams in Linguistics
}

\author{
Hans Smessaert ${ }^{1}$ and Lorenz Demey ${ }^{2}$ \\ 1 Department of Linguistics, KU Leuven \\ Hans. Smessaert@arts . kuleuven. be \\ 2 Center for Logic and Analytic Philosophy, KU Leuven; FWO \\ Lorenz.Demey@hiw.kuleuven. be
}

\begin{abstract}
The aim of this paper is to lay out the foundations of a typology of diagrams in linguistics. We draw a distinction between linguistic parameters - concerning what information is being represented - and diagrammatic parameters - concerning how it is represented. The six binary linguistic parameters of the typology are: (i) mono- versus multilingual, (ii) static versus dynamic, (iii) mono- versus multimodular, (iv) object-level versus meta-level, (v) qualitative versus quantitative, and (vi) mono- versus interdisciplinary. The two diagrammatic parameters are (i) iconic/concrete versus symbolic/abstract representation and (ii) static versus dynamic representation. We briefly illustrate how different types of linguistic diagrams can be analysed in terms of the interaction between the linguistic and the diagrammatic parameters.
\end{abstract}

Keywords: typology, linguistics, modularity, object vs metalevel, iconic representation, symbolic representation

\section{Diagrams and Linguistics}

In order to put the present paper in a somewhat broader context, let us start off by briefly considering three different perspectives which the Diagrams research community has taken upon the relationship between diagrams on the one hand and language or linguistics on the other hand. The first perspective - namely Diagrams versus Linguistics - adopts a primarily 'negative' relation of contrast between the two. The second and third perspectives both establish 'positive' relations, but they differ from one another in terms of their directionality, namely Linguistics for Diagrams as opposed to Diagrams for Linguistics.

Diagrams versus Linguistics. At least since the seminal paper of Larkin and Simon [9, research into reasoning systems - at the interface of Logic, Cognitive Science and Artificial Intelligence - has been concerned with similarities and differences between reasoning based on diagrammatic or visual information and reasoning based on sentential, propositional or linguistic information [5]16]18 19. Also outside the context of reasoning research, diagrammatic information is often distinguished from, and opposed to, linguistic information, e.g. when diagrams are said to occupy an intermediate position in between 'description' and 'depiction' [11, or when the category of 'non-picture visuals' is 
further divided into 'linguistic forms' - such as text, tables or notations - and 'non-picture graphical forms' - such as diagrams, graphs, charts and maps [4].

Linguistics for Diagrams. One way of establishing a more positive relation between a diagrammatic and a linguistic perspective involves using concepts from the field of linguistics to study or explain properties of diagrams. The key idea of the research field on Visual Languages and Computation is precisely that graphical representations resemble (natural or formal) languages in having a vocabulary and a grammar. As Mackinlay put it, "graphical presentations are actually sentences of graphical languages that have precise syntactic and semantic definitions" [10. This concept of the 'grammar' or 'language' of graphics and visual design is worked out in great detail by Kress \& van Leeuwen [8] as well as Engelhardt [6]. Interestingly, the first two perspectives on the relationship between diagrams and linguistics are integrated in Howse et al. [7, where the linguistic concept of a type-token distinction is taken to play a bigger role in diagrammatic systems than in linguistic systems, thus arguing for a more fine-grained syntax for diagrammatic representations.

Diagrams for Linguistics. An alternative way of connecting diagrams and linguistics proceeds by taking concepts from the field of diagrams research in order to study the visual representations used in linguistics to describe the properties of natural language expressions. Judging from the contributions to the proceedings of the nine Diagrams Conferences (2000-2016), this third perspective has received surprisingly little attention. The ones that do occur, however, testify of the fact that diagrams show up in various areas of linguistic research, ranging from phonological features [14, over tree representations for syntactic structures [3], to scales and sets for the semantic representation of tenses [2] and quantifiers [15.

Aim of the paper. The aim of the present paper is precisely to contribute to this third perspective, by laying out the foundations of a typology of diagrams in linguistics. To the best of our knowledge, such a typology has not been proposed so far. We draw a distinction between linguistic parameters in Section 2 concerning what information is being represented - and diagrammatic parameters in Section 3 - concerning how that information is represented. In Section 4 we briefly illustrate how different types of linguistic diagrams can be analysed in terms of the interaction between linguistic and diagrammatic parameters. In future work, the validity of the proposed parameters will be empirically tested by means of large-scale corpus research. In the long run, the resulting typology is hoped to contribute to the emerging field of philosophy of linguistics, and hence to the broader area of philosophy of science, in which the heuristic and didactic value of visualisation techniques is a well-established research topic [17.

\section{Linguistic Parameters}

As to the question of what information is being represented, the first four binary parameters of the typology receive an intrinsically linguistic characterisation, namely: (i) mono- versus multilingual, (ii) static versus dynamic, (iii) mono- 
versus multimodular, and (iv) object-level versus meta-level information. The remaining two binary parameters - (v) qualitative versus quantitative, and (vi) mono- versus interdisciplinary information - are of a more general nature.

Monolingual versus multilingual information. The first parameter distinguishes between the - MONOLINGUAL - study of 'language' and the - MULTILINGUAL - study of 'languages'. The study of language refers to expressions in a particular natural language - such as the simple English main clause The cat is sitting on the mat - on different levels of complexity. This complexity is traditionally related to the 'size' of the units under scrutiny, ranging from very small to very big, in particular from sounds over words and clauses to discourse. The study of languages, by contrast, is concerned with family relationships between (groups of) natural languages - such as the Germanic versus the Celtic language families. This field of study — often called 'linguistic typology' crucially involves the dimensions of space and time, since it aims to chart the geographical distribution of language families as well as their chronology, i.e. their genetic resemblance and descendance. Both the monolingual analysis of natural language expressions and the multilingual classification and comparison of language families very often make use of visual representations of various kinds.

Static versus dynamic information. The second parameter - which concerns the opposition between STATIC and DYNAMIC information - can first of all straightforwardly be connected to the linguistic contrast between synchrony and diachrony. The synchronic perspective considers the contemporary situation both 'internally', for any given individual language, and 'externally', for language families as a whole. The diachronic perspective, by contrast, investigates the historical changes and evolutions, again both language-internally and on the level of entire language families. It is important to stress, however, that the distinction between static and dynamic information is not restricted to the synchronic versus diachronic perspectives in linguistics. In general, static information concerns a stable situation or state of an object or concept, whereas dynamic information concerns processes, i.e. temporal or structural changes in the object or concept. For instance, in theoretical frameworks which assume that certain components of a natural language expression are moved to different positions in the structure.

Monomodular versus multimodular information. As already hinted at above, natural language expressions can be analysed on different levels, depending on the size of the units or components under investigation. Standardly, the following six linguistic modules are distinguished:

phonetics the articulation, acoustics and perception of speech sounds

phonology abstract sound segments, syllables and prosody

morphology the internal structure of words (derivation, compounding)

syntax sentence structure and word-order

semantics the meaning of words (lexical) and sentences (propositional)

pragmatics speech acts, interaction in conversation, discourse structure

According to the third parameter, MONOMODULAR information restricts the focus to properties, relations or concepts within one of the six modules above, 
whereas MULTIMODULAR information is at issue as soon as properties, relations or concepts from at least two modules are shown to interact in the representations.

Object-level versus meta-level information. As a fourth parameter, we propose a binary opposition between object-level information and meta-level information. In linguistics, the key 'objects' of investigation are natural language expressions: sounds, syllables, words, constituents, clauses, conversations and so on. Visual representations that explicitly contain (components of) such natural language expressions and their properties will be called OBJECT-LEVEL diagrams. Quite often, however, visual representations are concerned with properties of, or relations between, the linguistic concepts or the applied terminology, irrespective of any concrete natural language expression. Such representations will be called META-LEVEL diagrams.

Qualitative versus quantitative information. With the fifth parameter - which relates to the opposition between qualitative and quantitative information — we reach a more general, no longer intrinsically linguistic level. On the one hand, the analysis of properties of natural language expressions (object-level) or relations between linguistic concepts (meta-level) often yields QUALITATIVE or non-numerical data/information, concerning — for instance - linear ordering relations or hierarchical structures. On the other hand, linguistic analyses very often also generate QUANTITATIVE information, in the form of numerical values for certain parameters or attributes (or any other statistical properties). The latter receive visual representations such as tables or charts (bar charts, line charts, pie charts and so on) which are omnipresent in the scientific literature, but not specifically linguistic in nature [13, p. 61].

Monodisciplinary versus interdisciplinary information. As is the case in so many scientific areas, the field of linguistics interacts with a whole range of neighbouring disciplines, such as sociology, psychology, neuroscience, computer science (among many others), thus giving rise to the corresponding interdisciplinary fields of sociolinguistics, psycholinguistics, neurolinguistics, and computational linguistics. The sixth and final linguistic parameter therefore distinguishes between MONODISCIPLINARY information on the one hand - i.e. 'purely linguistic' information restricted to the core modules (or levels) for the analysis of linguistic expressions - and INTERDISCIPLINARY information on the other hand. After all, since those many interdisciplinary fields not only borrow concepts and methods from their non-linguistic source disciplines, but very often also adopt the corresponding visualisation strategies, we will need to take visualisations of 'mixed origin' into account as well.

\section{Diagrammatic Parameters}

Following the characterisation by Purchase [13, p. 59], a DIAGRAM is taken to be "a composite set of marks or visual elements - including lines, geometric shapes and individual words - on a two-dimensional plane, that - when taken together - represent a concept or object in the mind of the viewer. Diagrams are 
meant to depict appearance, structure, or workings of something, and are usually employed to support viewers' tasks, such as learning, designing, communicating, or simply understanding the concept depicted."

As a number of overview papers have demonstrated [1]13, it is notoriously difficult to provide an adequate set of visual principles for setting up a typology of diagrams. Nevertheless, as was the case in the previous section, a number of binary oppositions turn out to play a crucial role. In order to answer the question of how the linguistic information is being represented visually, two diagrammatic parameters are taken to underlie the typology. The main diagrammatic parameter is the classical, semiotic contrast between iconic/concrete and symbolic/abstract representations. On a secondary level, we draw a further distinction between static and dynamic representations.

Iconic versus symbolic representation. The central diagrammatic parameter to underlie the envisaged typology, is taken from the field of semiotics (the science of signs), namely the distinction between two major classes of signs - ICONS and SYMBOLS - which basically corresponds to the opposition between concrete and abstract representations [13, p. 59]. A diagram is called ICONIC or CONCRETE if there is a direct perceptual relationship of similarity or resemblance between the sign - i.e. the diagram or representation - and the referent i.e. the object being represented. A diagram is called SYMBOLIC or ABSTRACT if the relationship between the sign and the referent is purely arbitrary, based on sets of conventions within a given community.

ICONIC diagrams depict their objects in a form similar to their physical attributes or depict physical positional relationships between objects [13, p. 60]. Typical examples are anatomic illustrations, maps, or seating arrangements. Symbolic diagrams, by contrast, have no perceptual relationship to the concepts that they represent. Three broad categories can be distinguished [13, p. 60]: (i) graphs use geometric shapes to represent objects, and lines to depict relationships between objects, (ii) set diagrams use overlapping geometric shapes to depict set membership, and (iii) charts - as mentioned above — present numerical or quantitative information 1 . Note that specific diagrams may be of a composite type in that they combine notational properties of different (abstract and/or concrete) diagram subtypes [13, p. 61].

Within the symbolic category of graphs, the subtype of trees - think of the classical genealogical or family tree - deserves special mention here. The notion of hierarchical structure obviously plays a crucial role in many scientific disciplines [12 20. Also in the field of linguistics, trees pop up all over the place, first of all in the genealogical sense, as representations of the internal structure of language families. Secondly, also on the level of language - i.e. the properties

\footnotetext{
${ }^{1}$ In the field of Information Graphics or Data Visualisation, the different ways in which information can be structured have been captured under the acronym LATCH (= Location Alphabet Time Category Hierarchy), according to whether the elements are organised spatially, organised alphabetically, organised against a time line, divided into classes or ranked in order of priority 21. The Location dimension typically yields concrete diagrams, whereas the others standardly yield abstract diagrams.
} 
of concrete natural language expressions - the idea of hierarchical organisation is absolutely essential, with varying sizes for the units of analysis, depending on the particular linguistic module under consideration.

Static versus dynamic representation. Independently of the above opposition between iconic and symbolic diagrams, we will also distinguish between STATIC diagrams and DYNAMIC diagrams [13, p. 62]. A DYNAMIC diagram represents a succession or sequence of states or processes, either as a series of individual diagram $\$ 2$, or by using graphical elements such as arrows. By contrast, a STATIC diagram contains no graphical elements representing a succession or sequence. Note that it is possible for a static diagram to represent dynamic information, as long as it does not contain any dedicated graphical elements for this purpose (such as arrows). However, such a mismatch between the diagram and the information that it represents typically has a negative effect on the quality of the visual representation.

\section{Illustrating and applying the parameters}

In this section we briefly illustrate how different types of linguistic diagrams can be analysed in terms of the interaction between the linguistic parameters (LP) from Section 2 and the diagrammatic parameters (DP) from Section 3. Figure 1(a) iconically represents the tongue movement in the vowel space of the two dipthong sounds in Dutch words such as bruin ('brown') and koud ('cold'), whereas the spectrogram (from acoustics/physics) in Figure 1(b) provides quantitative information on the distribution of frequency over time (with intensity as gray scale), when pronouncing the Dutch expression aja ('yes indeed'). Figure 1(c) represents the historical development of the Insular branch of the Celtic language family, and Figure 1(d) represents the multimodular mismatches between the syntactic and morphological structures of the West Flemish subclause ...da-k eet- $n$ ('... that I am eating'). In Table 1 , the linguistic and diagrammatic parameters proposed above, are applied to the four linguistic diagrams from Figure 1. Notice that the genealogical tree structure in Figure 1(c) is a typical example of dynamic information (viz. the evolution of certain languages over time) being represented by means of a static diagram.

\section{Conclusions and Future Work}

In this paper we have laid out the foundations of a typology of diagrams in linguistics by looking at the interaction between six linguistic parameters and two diagrammatic parameters. In future work, the validity of the proposed parameters will be empirically tested by means of large-scale corpus research, based on a broad range of general linguistics journals and handbooks dedicated to the various linguistic modules. This will allow us to check whether the proposed

\footnotetext{
${ }^{2}$ Notice that such a series of diagrams can develop as an animation through time, or by juxtaposition in space.
} 

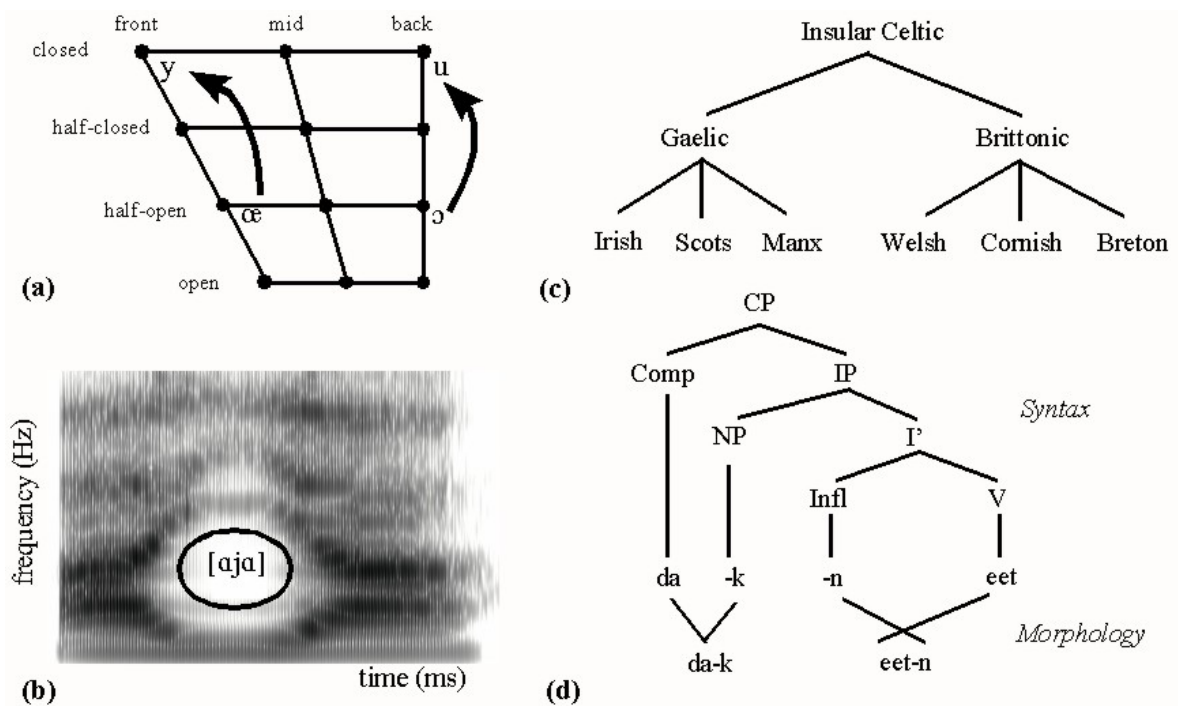

(c)

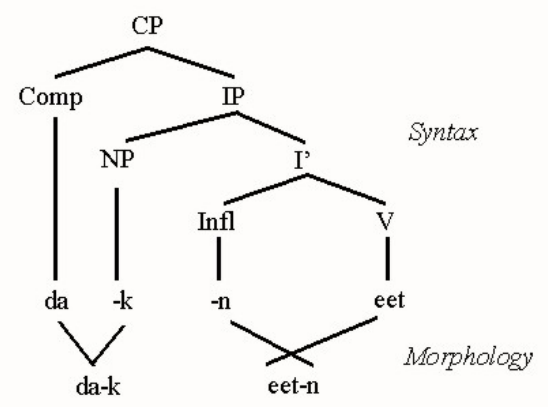

Fig. 1. Diagrams in Linguistics

\begin{tabular}{c|cc|cccc} 
& {$[-]$} & {$[+]$} & (a) & (b) & (c) & (d) \\
\hline \multirow{4}{*}{ LP } & monolingual & multilingual & - & - & + & - \\
& static & dynamic & + & + & + & - \\
& monomodular & multimodular & - & - & - & + \\
& object-level & meta-level & - & - & + & - \\
& qualitative & quantitative & - & + & - & - \\
& monodisciplinary & interdisciplinary & - & + & - & - \\
\hline \multirow{2}{*}{ DP } & iconic & symbolic & - & + & + & + \\
& static & dynamic & + & + & - & -
\end{tabular}

Table 1. Linguistic and diagrammatic parameters applied to Figure 1

parameters are sufficiently fine-grained to capture and classify all actually occurring diagrams, or whether further modifications are necessary. Ultimately, the resulting typology will help to clarify the heuristic and didactic value of visualisation techniques in linguistics, thus contributing to the emerging field of philosophy of linguistics.

\section{References}

1. Blackwell, A., Engelhardt, Y.: A meta-taxonomy for diagram research. In: Anderson, M., Meyer, B., Olivier, P. (eds.) Diagrammatic Representation and Reasoning, pp. 47-64. Springer (2002) 
2. Bosveld-de Smet, L.: Diagrams in second or foreign language learning??! In: BarkerPlummer, D., Cox, R., Swoboda, N. (eds.) Diagrams 2006, pp. 151-153. LNCS 4045, Springer (2006)

3. Bosveld-de Smet, L., de Vries, M.: Visualizing non-subordination and multidominance in tree diagrams: Testing five syntax tree variants. In: Stapleton, G., Howse, J., Lee, J. (eds.) Diagrams 2008, pp. 308-320. LNCS 5223, Springer (2008)

4. Cox, R.: Recognising, knowing and naming: Can object picture processing models accommodate non-picture visuals? In: Dwyer, T., Purchase, H., Delaney, A. (eds.) Diagrams 2014, pp. 161-175. LNCS 8578, Springer (2014)

5. Cox, R., Dale, R., Etchemendy, J., Barker-Plummer, D.: Graphical revelations: Comparing students' translation errors in graphics and logic. In: Stapleton, G., Howse, J., Lee, J. (eds.) Diagrams 2008, pp. 257-265. LNCS 5223, Springer (2008)

6. Engelhardt, Y.: The Language of Graphics: A Framework for the Analysis of Syntax and Meaning in Maps, Charts and Diagrams. Ph.D. thesis, Universiteit van Amsterdam, Institute for Logic, Language and Computation (2002)

7. Howse, J., Molina, F., Shin, S.J., Taylor, J.: On diagram tokens and types. In: Hegarty, M., Meyer, B., Narayanan, N.H. (eds.) Diagrams 2002, pp. 146-160. LNCS 2317, Springer (2002)

8. Kress, G.R., van Leeuwen, T.: Reading Images: The Grammar of Visual Design. Routledge (1996)

9. Larkin, J.H., Simon, H.A.: Why a diagram is (sometimes) worth ten thousand words. Cognitive Science 11(1), 65-100 (1987)

10. Mackinlay, J.D.: Automating the design of graphical presentations. ACM Transactions on Graphics 5(2), 110141 (1986)

11. Norman, J.: Differentiating diagrams: A new approach. In: Anderson, M., Cheng, P., Haarslev, V. (eds.) Diagrams 2000, pp. 105-116. LNCS 1889, Springer (2000)

12. Novick, L.R.: The importance of both diagrammatic conventions and domainspecific knowledge for diagram literacy in science: The hierarchy as an illustrative case. In: Barker-Plummer, D., Cox, R., Swoboda, N. (eds.) Diagrams 2006, pp. 1-11. LNCS 4045, Springer (2006)

13. Purchase, H.C.: Twelve years of diagrams research. Journal of Visual Languages and Computing 25(2), 57-75. (2014)

14. Salting, D.: Feature diagrams in phonology. In: Blackwell, A.F., Marriott, K., Shimojima, A. (eds.) Diagrams 2004, pp. 398-401. LNCS 2980, Springer (2004)

15. Sato, Y., Mineshima, K.: Human reasoning with proportional quantifiers and its support by diagrams. In: Jamnik, M., Uesaka, Y., Elzer Schwartz, S. (eds.) Diagrams 2016, pp. 123-138. LNCS 9781, Springer (2016)

16. Sato, Y., Mineshima, K., Takemura, R.: The efficacy of Euler and Venn diagrams in deductive reasoning: Empirical findings. In: Goel, A.K., Jamnik, M., Narayanan, N.H. (eds.) Diagrams 2010, pp. 6-22. LNCS 6170, Springer (2010)

17. Scholz, B.C., Pelletier, F.J., Pullum, G.K.: Philosophy of linguistics. In: Zalta, E.N. (ed.) The Stanford Encyclopedia of Philosophy. Stanford University (2016)

18. Shimojima, A.: The graphic linguistic distinction. Artificial Intelligence Review 13(4), 313-335 (1999)

19. Stenning, K., Oberlander, J.: A cognitive theory of graphical and linguistic reasoning: Logic and implementation. Cognitive Science 19, 97-140 (1995)

20. Tversky, B., Corter, J.E., Yu, L., Mason, D.L., Nickerson, J.V.: Representing category and continuum: Visualizing thought. In: Cox, P., Plimmer, B., Rodgers, P. (eds.) Diagrams 2012, pp. 23-34. LNCS 7352, Springer (2012)

21. Wurman, R.S.: Information Architects. Graphis Press Corporation (1996) 